\title{
Ventilator-associated pneumonia diagnosis: a prioritization exercise based on multi-criteria decision analysis
}

\author{
João Ferreira-Coimbra ${ }^{1}$ - Carmen Ardanuy ${ }^{2} \cdot$ Emili Diaz $^{3} \cdot$ Marc Leone $^{4} \cdot$ Gennaro De Pascale $^{5,6} \cdot$ Pedro Póvoa ${ }^{7,8,9}$. \\ Cristina Prat-Aymerich ${ }^{10} \cdot$ Ricardo Serrano-Garcia $^{11,12} \cdot$ Jordi Solé-Violan $^{13,14} \cdot$ Rafael Zaragoza $^{15} \cdot$ Jordi Rello $^{16}$
}

Received: 16 August 2019 / Accepted: 20 September 2019 / Published online: 25 October 2019

(C) Springer-Verlag GmbH Germany, part of Springer Nature 2019

\begin{abstract}
The aim was to provide global experts ranking on priorities in diagnostic tools for VAP in clinical practice. A multiple criteria decision analysis (MCDA) was performed to identify diagnosis tools for VAP diagnosis. Priority factors were identified after literature review. An international, multidisciplinary expert panel reviewed variables and ranked diagnostic tools. Experts from ten European hospitals participated. Regarding bedside clinical practices, seven required chest X-ray use in all patients, whereas six reported the use of blood cultures and endotracheal aspirate in all patients. Invasive techniques were routinely performed in seven sites. CRP, PCT, and Gram stains were performed in all patients by 5, 2, and 8, respectively. Impact on patient outcomes, safety, and impact on the decision to start antibiotic therapy were ranked as the top three relevant concerns $(7.7 / 10,7 / 10$, and 6.9/ 10, respectively). Chest X-ray was ranked as the most important imaging technique to diagnose VAP (score 251.7). Apart from blood cultures, endotracheal aspirate culture was identified as the main collection method for the microbiological testing (scores of 274.8 and 246.8, respectively). Mini-BAL was the preferred invasive technique with a score of 208. Top three biomarkers were CRP (score 184.3), PCT (181.3), and WBC (166.4). Gram stain (192.5) was prioritized among laboratory diagnostic techniques. Using MCDA, it is recommended to perform a combination of diagnostic techniques including images (chest X-ray), culture of clinical specimens (blood cultures and endotracheal aspirate), and biomarkers (CRP or PCT) for VAP diagnosis at the bedside. Gram stain was ranked as the preferred laboratory technique.
\end{abstract}

Keywords VAP $\cdot$ Diagnosis $\cdot$ MCDA $\cdot$ Multicriteria decision analysis $\cdot$ Ventilator-associated pneumonia $\cdot$ Microbiology diagnosis $\cdot$ Biomarkers $\cdot$ Imaging

\section{Introduction}

Ventilator-associated pneumonia (VAP) is a chest infection occurring $48 \mathrm{~h}$ after the initiation of mechanical ventilation. It is responsible for increasing hospital length of stay and costs $[1,2]$. Many efforts are still being performed to reduce their burden, especially using prevention bundles [3, 4], which have been shown to be effective in reducing its rate. VAP is a continuous process presenting with nonspecific clinical features [5], with some limitations regarding its definition and

Electronic supplementary material The online version of this article (https://doi.org/10.1007/s10096-019-03720-x) contains supplementary material, which is available to authorized users.

João Ferreira-Coimbra

joaoferreiracoimbra@gmail.com

Extended author information available on the last page of the article diagnosis, because gold-standard tests are still lacking. It is also missing clear evidence to prioritize diagnostic tests and how they impact clinical outcomes. As evidence is weak, expert opinion should be taken into account. Multi-criteria decision analysis (MCDA) is widely used in decision-making in various areas in order to rank priorities and is also used in medicine with the same purpose putting together evidence and expert opinion $[6,7]$.

Our aim is to create a set of priorities of tools for VAP diagnosis in adult patients using a MCDA. Furthermore, we also want to provide data regarding experts' preferences and the use of diagnostic tools in their practice.

\section{Methods}

An international panel of experts with a background in intensive care medicine and clinical microbiology was directly 
invited by email to participate in this study by filling an electronic survey (Supplementary material 1). Participants were chosen by study coordinator (JR), according to their expertise in clinical practice, participation in clinical studies, and publications. Experts also contributed to article revision and provision of the final version.

A comprehensive list of VAP diagnostic tests and priority criteria included in multi-criteria decision analysis (MCDA, methodology described elsewhere [8]) were chosen by the study coordinator after literature review [9-11]. Seven priority criteria were defined by authors (JFC and JR) and independently weighted by the panel, according to their relative importance to each other. Diagnostic tests were evaluated individually against priority criteria, by each expert. These results were then multiplied by the weight of each priority criterion to obtain individual scores for each diagnostic test. Then, sum score was obtained after addition of all the partial scores previously achieved. Each variable was transformed into a $0-10$ scale in order to facilitate their comparison. All responses are categorical variables since they are reported using descriptive statistics (proportions and percentages). Median and interquartile range (IQR) were used to analyze nonparametric variables.

Panel members were also inquired about their perceived relevance of each diagnostic tool and also about their use in clinical practice. For the purpose of this study, VAP is defined as a chest infection that occurs after $48 \mathrm{~h}$ of endotracheal tube placement $[9,10]$. This study focuses on adult patients. Due to the lack of patients, data in this study, ethical approval, and informed consent were waived.

\section{Results}

A panel of ten European experts with background in intensive care medicine and clinical microbiology accepted to participate in our study.

Seven in 10 experts confirmed the use of chest X-ray in all patients with VAP, while 5/10 and 4/10 admitted the use of chest $\mathrm{CT}$ and lung ultrasound in more than $50 \%$ of patients, respectively. The whole group used at least one imaging technique for VAP diagnosis. Blood cultures and endotracheal aspirate were declared to be the most used methods to obtain microbiological specimens for culture. All group members reported their use in more than $60 \%$ and $50 \%$ of cases, respectively. Any invasive technique is used in more than $50 \%$ of VAP patients by 7 experts, while bronchoalveolar lavage is the most commonly used technique $(5 / 10$ reported its use in more than $50 \%$ of patients). Transbronchial biopsy was reported as the least used bronchoscopy technique. All declined the use of IL6 and IL10, as well as pro-adrenomedulin in VAP diagnosis in their practice. White blood cell count (WBC), C-reactive protein (CRP), and procalcitonin (PCT) are used in all patients by $7 / 10,5 / 10$, and $2 / 10$ members of the panel, respectively.
Two $(2 / 10)$ reported the absence of the use of biomarkers in $10-20 \%$ of patients, while $3 / 10$ reported no use of CRP and PCT. Gram stain and MALDI-TOF (matrix assisted laser desorption ionization-time of flight) were reported as the most used among microbiological techniques, performed in all patients by $8 / 10$ and $6 / 10$ physicians, respectively.

After an MCDA, impact in patient outcomes (7.7/10), risk to the patient (7.0/10), and impact on decision to start antibiotic therapy $(6.9 / 10)$ were ranked as the major concerns for VAP diagnosis, followed by time to clinical relevant result (6.0/10), test availability (5.7/10), need for professional experience (4.0/10), and cost (2.7/10).

Chest X-ray was ranked as the most important imaging technique (score 251.7). "None imaging technique use" strategy obtained the least score (143.6). Blood cultures and endotracheal aspirate culture were scored as the most important microbiological cultures (scores 274.8 and 246.8). Among invasive techniques, mini-bronchoalveolar lavage (miniBAL) was ranked first (208.0), followed by bronchoalveolar lavage (BAL; 204.9), the telescopic catheter (176.7), and transbronchial biopsy (78.7). Among these techniques, miniBAL was intended as the safest (score 46.7) and BAL the riskier (after transbronchial biopsy, that is rarely used). Meanwhile, the same distribution appeared in need of professionals' experience, where mini-BAL was ranked as the one that needs less experience (Table 1).

CRP (score 184.3), PCT (score 171.3), and WBC (score 166.4) were ordered as the most important biomarkers in VAP diagnosis. Gram stain had the most favorable score in microbiological techniques (score 192.5), followed by MALDI-TOF (score 180.0), PCR tests (score 177.5), and Gene Expert ${ }^{\circledR}$ for ESBL (score 162.9). Regarding time to clinical relevant result, Gram stain, PCR tests, and MALDITOF were top scored. Table 1 shows the overall results of the MCDA analysis. According to panel members ranking, chest $\mathrm{CT}$ is the imaging technique that impacts the strongest on patient outcomes. Blood cultures, MALDI-TOF, and PCR are the more important in test microbiology cultures and techniques, and PCT is intended as the biomarker with most impacting on patient outcomes. Gram stain, MALDITOF, and PCR tests were scored first in "Time to clinical relevant result". Bronchoscopy techniques (included transbronchial biopsy) were considered as the unsafest for VAP diagnosis (Table 1).

In this study survey, the panel was also asked to show their own relevance for each test (Table 2 shows, in a 10 value scale, expert preference in VAP diagnosis). Chest $\mathrm{X}$-ray was the preferred imaging technique (score 8.5/ 10), bronchoalveolar lavage culture the preferred away to assess lung pathogens (score 8.0/10). Procalcitonin (score 8.4/10) and CRP (score 8.0/10) were classified as the most relevant biomarkers. Gram stain $(8.3 / 10)$ was the preferred microbiological technique. 
Table 1 MCDA results

\begin{tabular}{|c|c|c|c|c|c|c|c|c|}
\hline & $\begin{array}{l}\text { Impact on } \\
\text { patient } \\
\text { outcomes }\end{array}$ & $\begin{array}{l}\text { Risk to the } \\
\text { patient }\end{array}$ & $\begin{array}{l}\text { Impact on decision } \\
\text { to start antibiotic } \\
\text { therapy }\end{array}$ & $\begin{array}{l}\text { Time to } \\
\text { clinical } \\
\text { relevant } \\
\text { result }\end{array}$ & $\begin{array}{l}\text { Test } \\
\text { availability }\end{array}$ & $\begin{array}{l}\text { Need of } \\
\text { professionals } \\
\text { experience }\end{array}$ & Cost & $\begin{array}{l}\text { Sum } \\
\text { score }\end{array}$ \\
\hline Weight & 7.7 & 7 & 6.9 & 6 & 5.7 & 4 & 2.7 & \\
\hline \multicolumn{9}{|l|}{ Imaging techniques } \\
\hline X-ray & 55.8 & 49 & 53.5 & & 54.2 & 23 & 16.2 & 251.7 \\
\hline Chest CT & 69.3 & 47.3 & 55.2 & & 32.8 & 20 & 6.8 & 231.4 \\
\hline Lung ultrasound & 48.1 & 56 & 46.6 & & 37 & 17 & 18.2 & 222.9 \\
\hline None & 18.8 & 22.8 & 17.2 & & 18.5 & 40 & 26.3 & 143.6 \\
\hline \multicolumn{9}{|l|}{ Microbiological tests } \\
\hline Blood cultures & 57.8 & 67.7 & 50.6 & & 49.4 & 30 & 19.3 & 274.8 \\
\hline Endotracheal aspirate culture & 33.4 & 59.5 & 42.6 & & 53.2 & 33.3 & 24.8 & 246.8 \\
\hline $\begin{array}{l}\text { Mini-bronchoalveolar lavage } \\
\text { culture }\end{array}$ & 47.5 & 46.7 & 40.3 & & 30.4 & 26 & 17.1 & 208 \\
\hline $\begin{array}{l}\text { Bronchoalveolar lavage } \\
\text { culture }\end{array}$ & 53.9 & 28 & 54.1 & & 31.4 & 22.6 & 14.9 & 204.9 \\
\hline Telescopic catheter & 51.3 & 31 & 35.7 & & 25.7 & 20 & 13 & 176.7 \\
\hline Transbronchial biopsy & 25.7 & 11.7 & 18.4 & & 9.5 & 8 & 5.4 & 78.7 \\
\hline \multicolumn{9}{|l|}{ Biomarkers } \\
\hline CRP & 63.8 & & 53.2 & & 48 & & 19.3 & 184.3 \\
\hline PCT & 66 & & 57.2 & & 41.5 & & 16.6 & 181.3 \\
\hline WBC & 40.7 & & 53.2 & & 52.1 & & 20.4 & 166.4 \\
\hline Pro-adrenomedullin & 47.3 & & 27.6 & & 21.2 & & 10 & 106.1 \\
\hline None & 17.6 & & 33.5 & & 27.7 & & 23.9 & 102.7 \\
\hline IL6 & 39.6 & & 28.6 & & 18.7 & & 9.6 & 96.5 \\
\hline IL10 & 33 & & 22.7 & & 18.7 & & 8.1 & 82.5 \\
\hline \multicolumn{9}{|l|}{ DIAGNOSTIC techniques } \\
\hline Gram stain & 52.9 & & & 51 & 49.2 & 17.5 & 21.9 & 192.5 \\
\hline MALDI-TOF & 59.7 & & & 42.7 & 46.3 & 18.5 & 12.8 & 180 \\
\hline PCR tests & 59.7 & & & 45.8 & 39.2 & 23 & 9.8 & 177.5 \\
\hline Gene Expert ${ }^{\circledR}$ for ESBL & 56.8 & & & 39.8 & 32.8 & 24 & 9.5 & 162.9 \\
\hline Multiplex PCR & 52 & & & 34.5 & 28.5 & 22 & 9.8 & 146.8 \\
\hline Serological tests & 25 & & & 21.7 & 29.9 & 25 & 16.5 & 118.1 \\
\hline Rapid automated microscopy & 29.8 & & & 27 & 15.7 & 19 & 15.5 & 107 \\
\hline None & 2.9 & & & 7.5 & 15 & 31 & 25.6 & 82 \\
\hline
\end{tabular}

\section{Discussion}

This study represents the first comprehensive recommendations for VAP diagnosis, enclosing image, microbiologic specimens, biomarkers, and lab tests using a formal decision support tool. According to this, VAP diagnosis should be based on chest X-ray, blood cultures, endotracheal aspirate, CRP (or PCT), and Gram stain.

The clinical diagnostic criteria for VAP are subjective, lack specificity. For that, the use of diagnostic tools is widely variable according to local practices and different beliefs of its importance [12]. We evaluated some priority factors that can affect the diagnostic approach. Impact on patient outcomes and risk were the characteristics of major concern to experts, revealing concerns with final patient results and also the importance of risk evaluation before diagnostic tools use. Time to clinical relevant result is also a key decision factor, which had only been tested for microbiological techniques, in order to rank these techniques as they are critical to assess rapidly deteriorating patients. Surprisingly, in an era dominated by health economics and healthcare resource limitation, experts stated the cost as the least important characteristic, in spite of being an important limitation in tool availability.

Chest X-ray is the preferred imaging method for VAP diagnosis in this study. No expert stated to bypass an imaging method to diagnose VAP in clinical practice, in contrast to the CDC approach $[13,14]$, which excludes any imaging method for ventilator-associated events (VAE) surveillance. Our results 
Table 2 Experts panel preference in VAP diagnosis (in proportion 0-10)

\begin{tabular}{|c|c|c|c|c|c|c|c|}
\hline \multicolumn{2}{|l|}{ Imaging techniques } & \multicolumn{2}{|l|}{ Microbiological tests } & \multicolumn{2}{|l|}{ Biomarkers } & \multicolumn{2}{|l|}{ Other laboratory techniques } \\
\hline Chest X-ray & 8.5 & BAL culture & 8.0 & PCT & 8.4 & Gram stain & 8.3 \\
\hline Chest CT & 7.8 & Mini-BAL culture & 7.0 & CRP & 8.0 & MALDI-TOF & 7.5 \\
\hline Lung ultrasound & 6.3 & ET aspirate & 5.8 & WBC & 6.3 & Gene Expert® & 6.8 \\
\hline \multirow[t]{5}{*}{ None } & 2.5 & Blood cultures & 5.3 & Pro-ADM & 5.0 & Multiplex PCR & 6.5 \\
\hline & & Telescopic catheter & 6.3 & None & 4.7 & PCR tests & 6.1 \\
\hline & & Transbronchial biopsy & 2.5 & IL6 & 3.9 & Rapid automated microscopy & 4.8 \\
\hline & & & & IL 10 & 3.7 & Serological tests & 3.9 \\
\hline & & & & & & None & 1.3 \\
\hline
\end{tabular}

$B A L$ bronchoalveolar lavage, ET aspirate endotracheal aspirate, $P r o-A D M I$ pro-adrenomedullin

are in line with the ECDC strategy [15], which preserves chest imaging as a key for VAP surveillance and also with studies showing different prognosis of VAP and ventilator-associated tracheobronchitis [16]. In spite of this controversy, it is pertinent to keep in mind that VAP surveillance and diagnosis or clinical management are different approaches. Important to note that, despite lung ultrasound (LUS) use is increasing [17], in our study experts ranked as the least important imaging technique. This could be explained by the lack of training and access in LUS, which can limit its use.

In IDSA/ATS 2016 [10] and ERS/ESICM/ESCMID/ ALAT 2017 [9] guidelines, biomarkers used are not supported in VAP diagnosis as well as in decision for antibiotic withdrawal (except in specific circumstances). Meanwhile, biomarkers are abundantly used worldwide in VAP diagnosis, despite clear evidence proving that their positive impact on outcomes had never been published [18]. Most of our panel declared the use of WBC, CRP, and PCT in clinical practice, in spite of MCDA showed a preference by CRP and PCT. This could be explained by their low cost, and also, their use is generally intended as a good clinical practice (as some behaviors in medicine that are not supported by high-quality evidence). Differently, IL6, IL10, and Pro-adrenomedullin remain usefulness tools beyond investigational purposes.

Microbiological cultures and tests remain one of the most divergent issues in different guidelines [9-11]. Blood cultures, despite infrequently positive, were previously recognized as important tools in VAP [19], since bacteremic VAP is associated with higher mortality [20]. Although, in our MCDA experts ranked blood cultures as the most important culture specimen, when asked about their preferences in VAP diagnosis, blood cultures were only the fourth preferred. Between the other methods of specimens' collection for culture, endotracheal aspirate score first in MCDA, at the same time as BAL and mini-BAL were the preferred (by this order) invasive techniques. This can be related to the controversy about this issue in international guidelines. While American guidelines [10] recommend noninvasive and semi-quantitative sampling, European guidelines [9] recommend invasive and quantitative sampling. Interestingly, both are qualified as "Weak recommendation" and "Low-quality evidence", exposing that this concerning issue recommendation is more associated with divergent interpretation than different recognized evidence.

On the other hand, in the MCDA, mini-BAL scored slightly better than BAL, mainly driven by risk profile (safer) and less necessity for professionals' experience. This represents an important field, as in some places professionals lack experience in bronchoscopic BAL or if experienced operators are not always present. Besides cost and availability were out of the highest experts priorities, very specific tests as PCR tests and multiplex PCR were not in the top priorities regarding microbiological techniques. Timely results are of the highest importance in critically ill patient outcomes $[21,22]$ and also in reducing antibiotic pressure; therefore, the need for timely results is a relevant issue. In this subject, Gram stain, PCR tests, and Multiplex PCR that are able to produce results within 1-2 h can have a special role. Notwithstanding, it is also important to take into account local microbiology because tools like Gene Expert ${ }^{\circledR}$ for ESBL can be prioritized in some places.

This study has several limitations. First, the experts panel a limited European panel that can impair study generalization for other areas. Second, derived recommendations and data from patients did not take into account nor the number of patients treated in physicians ICUs, neither the physicians' experience. Third, specific patients admitted to each ICU (cardiac, burns, trauma, etc) were not addressed. Fourth, the availability of diagnostic tools in each hospital was not addressed, which could limit physicians' experience with some diagnostic techniques, influencing their beliefs.

The strengths include the study methodology (MCDA) and the possible generalization of this data, as previously reported [23], and the possibility to update this information in the future if new diagnostic tools becomes available in clinical practice.

Through MCDA analysis, this experts panel recommends for VAP diagnosis a set of chest X-ray, blood cultures, endotracheal aspirate, CRP (and PCT), and Gram stain. 


\section{References}

1. Safdar N, Dezfulian C, Collard HR, Saint S. Clinical and economic consequences of ventilator-associated pneumonia: A systematic review. Crit Care Med. 2005. https://doi.org/10.1097/01.CCM. 0000181731.53912.D9

2. Rello J, Ollendorf DA, Oster G, et al. Epidemiology and outcomes of ventilator-associated pneumonia in a large US database. Chest. 2002. https://doi.org/10.1378/chest.122.6.2115

3. Klompas M, Branson R, Eichenwald EC, et al. Strategies to Prevent Ventilator-Associated Pneumonia in Acute Care Hospitals: 2014 Update. Infect Control Hosp Epidemiol. 2014. https://doi.org/10. 1086/677144

4. Meduri GU. Diagnosis and differential diagnosis of ventilator-associated pneumonia. Clin Chest Med. 1995.

5. Fagon JY, Chastre J, Hance AJ, Domart Y, Trouillet JL, Gibert C. Evaluation of clinical judgment in the identification and treatment of nosocomial pneumonia in ventilated patients. Chest. 1993. https://doi.org/10.1378/chest.103.2.547

6. Rello J, Kalwaje Eshwara V, Lagunes L, et al. A global priority list of the TOp TEn resistant Microorganisms (TOTEM) study at intensive care: a prioritization exercise based on multi-criteria decision analysis. Eur J Clin Microbiol Infect Dis. 2019. https://doi.org/10. 1007/s10096-018-3428-y

7. Tacconelli E, Carrara E, Savoldi A, et al. Discovery, research, and development of new antibiotics: the WHO priority list of antibioticresistant bacteria and tuberculosis. Lancet Infect Dis. 2018. https:// doi.org/10.1016/S1473-3099(17)30753-3

8. Belton V, Stewart T (2002) Multiple Criteria Decision Analysis: An Integrated Approach. Kluwer Academic Publishers, Massachusetts https://doi.org/10.1007/978-1-4615-1495-4

9. Torres A, Niederman MS, Chastre J, et al. International ERS/ ESICM/ESCMID/ALAT guidelines for the management of hospital-acquired pneumonia and ventilator-associated pneumonia. Eur Respir J. 2017. https://doi.org/10.1183/13993003.00582-2017

10. Kalil, A. C., Metersky, M. L., Klompas, M., Muscedere, J., Sweeney, D. A., Palmer, L. B., ... Brozek JL. Management of Adults with Hospital-Acquired and Ventilator-Associated Pneumonia: 2016 Clinical Practice Guidelines by the Infectious Diseases Society of America and the American Thoracic Society. Infect Dis Clin Pract. 2016. https://doi.org/10.1097/IPC. 0000000000000476

11. Leone M, Bouadma L, Bouhemad B, et al. Hospital-acquired pneumonia in ICU. Anaesth Crit Care Pain Med. 2018. https://doi.org/ 10.1016/j.accpm.2017.11.006

12. Koulenti D, Lisboa T, Brun-Buisson C, et al. Spectrum of practice in the diagnosis of nosocomial pneumonia in patients requiring mechanical ventilation in European intensive care units. Crit Care Med. 2009. https://doi.org/10.1097/CCM.0b013e3181a037ac

13. Magill SS, Klompas M, Balk R, et al. Developing a new, national approach to surveillance for ventilator-associated events. Crit Care Med. 2013. https://doi.org/10.1097/CCM.0b013e3182a262db

14. Klompas M. Complications of Mechanical Ventilation - The CDC's New Surveillance Paradigm. N Engl J Med. 2013. https:// doi.org/10.1056/nejmp1300633

15. Plachouras D, Lepape A, Suetens C. ECDC definitions and methods for the surveillance of healthcare-associated infections in intensive care units. Intensive Care Med. 2018. https://doi.org/10. 1007/s00134-018-5113-0

16. Martin-Loeches I, Povoa P, Rodríguez A, et al. Incidence and prognosis of ventilator-associated tracheobronchitis (TAVeM): A multicentre, prospective, observational study. Lancet Respir Med. 2015. https://doi.org/10.1016/S2213-2600(15)00326-4

17. Wang G, Ji X, Xu Y, Xiang X. Lung ultrasound: A promising tool to monitor ventilator-associated pneumonia in critically ill patients. Crit Care. 2016. https://doi.org/10.1186/s13054-016-1487-y

18. Palazzo SJ, Simpson T, Schnapp L. Biomarkers for ventilator-associated pneumonia: Review of the literature. Hear Lung J Acute Crit Care. 2011. https://doi.org/10.1016/j.hrtlng.2010.11.003

19. Rello J, Gallego M, Mariscal D, Soñora R, Valles J. The value of routine microbial investigation in ventilator-associated pneumonia. Am J Respir Crit Care Med. 1997. https://doi.org/10.1164/ajrccm. 156.1.9607030

20. Agbaht K, Diaz E, Muñoz E, et al. Bacteremia in patients with ventilator-associated pneumonia is associated with increased mortality: A study comparing bacteremic vs. nonbacteremic ventilatorassociated pneumonia. Crit Care Med. 2007. https://doi.org/10. 1097/01.CCM.0000277042.31524.66

21. Kollef MH, Sherman G, Ward S, Fraser VJ. Inadequate antimicrobial treatment of infections: A risk factor for hospital mortality among critically III patients. Chest. 1999. https://doi.org/10.1378/ chest.115.2.462

22. Pulido MR, Moreno-Martínez P, González-Galán V, et al. Application of BioFire FilmArray Blood Culture Identification panel for rapid identification of the causative agents of ventilatorassociated pneumonia. Clin Microbiol Infect. 2018. https://doi.org/ 10.1016/j.cmi.2018.06.001

23. Rello J, Lode H, Cornaglia G, Masterton R. A European care bundle for prevention of ventilator-associated pneumonia. Intensive Care Med. 2010. https://doi.org/10.1007/s00134-010-1841-5

Publisher's note Springer Nature remains neutral with regard to jurisdictional claims in published maps and institutional affiliations.

\section{Affiliations}

\section{João Ferreira-Coimbra ${ }^{1}$ - Carmen Ardanuy ${ }^{2}$ - Emili Diaz ${ }^{3}$ - Marc Leone ${ }^{4} \cdot$ Gennaro De Pascale $^{5,6} \cdot$ Pedro Póvoa $^{7,8,9}$. Cristina Prat-Aymerich ${ }^{10} \cdot$ Ricardo Serrano-Garcia $^{11,12} \cdot$ Jordi Solé-Violan $^{13,14} \cdot$ Rafael Zaragoza $^{15} \cdot$ Jordi Rello $^{16}$}

1 Internal Medicina Department, Centro Hospitalar Universitário do Porto, Porto, Portugal

2 Microbiology Department, Hospital Universitari de Bellvitge, University of Barcelona, CIBERES, Instituto Salud Carlos III, Madrid, Spain

3 Department of Medicine, Universitat Autònoma de Barcelona (UAB), Critical Care Department, Corporació Sanitària Parc Taulí,
Sabadell, CIBERES Ciber de Enfermedades Respiratorias, Barcelona, Spain

4 Department of Anesthesiology and Critical Care Medicine, Aix Marseille University, Assistance Publique Hôpitaux de Marseille, Nord hospital, Marseille, France

5 Dipartimento di Scienza dell'Emergenza, Anestesiologiche e della Rianimazione-UOC di Anestesia, Rianimazione, Terapia Intensiva e 
Tossicologia Clinica, Fondazione Policlinico Universitario A. Gemelli IRCCS, Rome, Italy

6 Università Cattolica del Sacro Cuore, Istituto di Anestesia e Rianimazione, Rome, Italy

7 NOVA Medical School, New University of Lisbon, Lisbon, Portugal

8 Center for Clinical Epidemiology and Research Unit of Clinical Epidemiology, OUH Odense University Hospital, Odense, Denmark

9 Hospital de São Francisco Xavier, Centro Hospitalar de Lisboa Ocidental, Lisbon, Portugal

10 Hospital Universitari Germans Trias i Pujol, Fundació Institut d'Investigació en Ciències de la Salut Germans Trias i Pujol, Universitat Autònoma de Barcelona, CIBER Enfermedades Respiratorias, Barcelona, Spain
11 Intensive Medicine Service, General University Hospital of Alicante), Alicante, Spain

12 Department of Clinical Medicine, Miguel Hernández University of Elche, Alicante, Spain

13 Intensive Care Medicine Departament, Hospital Universitario de GC De Negrín, Las Palmas de GC, Spain

14 CIBERES, Instituto Salud Carlos III, Madrid, Spain

15 Intensive Care Unit, Hospital Universitario Dr. Peset, Valencia, Spain

16 Clinical Research/Epidemiology In Pneumonia \& Sepsis (CRIPS), Vall d'Hebron Institute of Research, CIBERES-Centro de Investigación en Red de Enfermedades Respiratorias, Barcelona, Spain 